\title{
High School Student's Knowledge, Attitude and Participation in Sexual Health Education in Rural Northern Ghana
}

\author{
Joshua Sumankuuro', Albert Asuuri², Maurice Danang Mikare², Frederick Ngmenkpieo3, \\ Judith Crockett ${ }^{1}$, Joseph K. Wulifan ${ }^{4}$ \\ ${ }^{1}$ School of Community Health, Charles Sturt University, NSW, Australia \\ ${ }^{2}$ Zuarungu Nursing \& Midwifery Training College, Bolgatanga, Ghana \\ ${ }^{3}$ Department of Education, University for Development Studies, Wa, Ghana \\ ${ }^{4}$ Department of Management Studies, School of Business and Law, University for Development Studies, Wa, Ghana \\ Email: joshsumankuuro@gmail.com
}

How to cite this paper: Sumankuuro, J., Asuuri, A., Mikare, M.D., Ngmenkpieo, F., Crockett, J. and Wulifan, J.K. (2020) High School Student's Knowledge, Attitude and Participation in Sexual Health Education in Rural Northern Ghana. Journal of Biosciences and Medicines, 8, 64-83.

https://doi.org/10.4236/jbm.2020.85007

Received: March 14, 2020

Accepted: May 3, 2020

Published: May 6, 2020

Copyright (๑) 2020 by author(s) and Scientific Research Publishing Inc. This work is licensed under the Creative Commons Attribution International License (CC BY 4.0).

http://creativecommons.org/licenses/by/4.0/

\begin{abstract}
Background: In Ghana, sex education has been a part of the post-independence school curriculum, aimed to fill a significant knowledge gap, prepare them to make healthy sexual choices. However, sex education is not apparent in the schools. We aimed to understand senior high school adolescents' knowledge, perspectives on contraceptive use, and attitude towards sexual health education in the Upper East Region of Ghana. Methods: This was a descriptive study involving 329 students (from 15 - 19) comprising males $(\mathrm{n}=166,50.5 \%)$ and females ( $n=163,49.5 \%$ ) from a senior high school in the Upper East Region of Ghana. Eighteen-point Likert scale items with four-point responses-strongly agree, agree, disagree, and strongly disagree-were used to measure students' perspectives in the Upper East Region, Ghana. Results: The results show that most students $(\mathrm{n}=150,45.6 \%)$ strongly disagreed with reporting first developmental changes to parents and teachers. For students who agree $(\mathrm{n}=101$, $30.7 \%)$, more males than females (55.4\% versus $44.6 \%$ ) favoured the recommendation. About $42 \%$ strongly disagreed with seeking professional counsel on contraceptive use, with more (52.6\%) females in support of non-use of the advice of health personnel. Similarly, most respondents sought information on sex education from peers and were more comfortable associating and sharing adolescent experiences than with parents and teachers. Conclusion: There is inadequate knowledge of the adolescent youth on sexual health issues. Therefore, we recommend that qualitative studies be conducted to understand the students' views on sexual and reproductive health knowledge and access to services.
\end{abstract}




\section{Keywords}

Adolescent Health, Sex Education, Contraception, Health Education, Ghana

\section{Background}

Adolescents worldwide are sexually active and tend to engage in potentially unhealthy sexual behavior. For example, the 2014 Ghana Demographic and Health Survey [1] indicates Adolescents aged 10 - 19 and young adults aged 20 - 24 together constitute $29.3 \%$ of Ghana's population and face particular challenges related to sexual and reproductive health, risks and vulnerabilities [2]. It is estimated that about 15 million adolescents aged 15 - 19 years give birth yearly, 4 million obtain an abortion, and about 100 million become infected with sexually transmitted diseases (STDs) annually [3] [4] [5]. Globally, about 40\% of all HIV/AIDS cases involve adolescents aged 15 - 20 years; an estimated 7000 youth are infected daily [6] [7] [8]. The 2011 UN Millennium Development Goals Report [9] has shown that nearly $23 \%$ of people living with HIV, globally, are under the age of 25 . Young people aged 15 to 20 accounts for $41 \%$ of new infections among those aged 15 and older, and women represent a slight majority (about 60\%) of people living with HIV in 2017 [8].

There are many reasons for the growing attention to the health of adolescents in Ghana. Adolescent sexual health education has transitioned from the National Adolescent Health and Development Programme in 2001 to the current medium-term Adolescent Health Service Policy and Strategy (2016-2020) [10]. Previous policy frameworks have engineered the reduction of HIV infections among adolescents aged 15 - 20 years decreased from 50\% to $30 \%$ in 2015. Another achievement was realised in respect of about $72 \%$ of females, aged below 20 years who reported having used skilled assistance during birth [2] [10]. In terms of family planning, only $5 \%$ and $17 \%$ of females aged $15-19$ and $20-24$, respectively received messages when they visited a health facility in 2015 [10]. All of these interventions aim to prepare the future generations for responsible living and ensure youth actively participate in the development discourse, provide them with information, education, and communication on reproductive and sexual health, prevention of early pregnancy, family planning, sexually transmitted infections prevention [11], achieving a decline in age at first sex, reducing rates of unsafe abortion, and education about sexual identities and relationships within the social context of the society, responsible sexual attitudes and behavior [12] [13].

Ghana has experienced a high growth rate of the youth population over the last two decades [14]. The early sexual maturation among females and males, together with a tendency for sexual activity to begin at younger ages than later, has increasingly placed adolescents at risk of sexually transmitted infections (STIs) such as HIV/AIDS [15] [16]. As is the case in many African communities, this early maturation occurs in the context of rapid social change that disrupts family life [5] 
[17] [18]. Hence, social and religious institutions, such as the extended family, the church, and mosques, which once governed values and rites of passage such as marriage, have been largely replaced by secular institutions [19] [20]. Notwithstanding the literature has shown that sexual matters are among popular topics for conversation and gossip [14], research also shows that a confluence of cultural, religious, and geographical factors creates a sensitive environment where issues of sexual and reproductive health have remained a taboo for decades [21] [22] [23]. For example, in Ghana, it is also culturally unwelcome to discuss sex and sexual issues with adolescents outside the family [24], including teachers and health professionals [18] [19] [22] [23]. Considering this, exposure of young adults to unsafe sex, drugs, and alcohol abuse seems to be on the increase [2] [10]. These practices constitute risky sexual behaviours accounting for the incidence of STIs among a more significant number of the youth [12] [16].

Sex education since post-independence in Ghana (1980) has always been dealt with in Ghana's educational system by integrating it into other subject areas within the framework of the School Health Programme (SHEP) [10] [25]. Relative to improving sexual behaviour, all schools, including senior High Schools (SHS), are supposed to have lessons related to sex and safe sex practices and encourage students to translate such lessons into practice.

Although Ghana has implemented adolescent sexual health education after independence, it appears few understand, respond to behavioural changes, and apply the relevant knowledge in their lives. Aside from this, little is known about the impacts of high school sexual health education on adolescents. Therefore, this study aimed to understand students' knowledge, attitude, and participation in sex education, as delivered in one secondary high school in Ghana; in so doing, it addresses the following questions:

\section{Study Objectives}

The study sought to:

1) Examine the knowledge level of students at a Senior High School on sex education.

2) Determine the usage of family planning methods/practices among students at the senior high school.

3) Assess the attitude of students at the senior high school towards sex education.

\section{Methods}

\subsection{Design}

The study was an exploratory, descriptive survey to assess the sexual behaviour of students of Zuarungu Senior High School. The descriptive design attempts to conclude findings to cover the target population and explaining what happens at a point in time. 


\subsection{Study Setting}

This study was carried out in a Senior High School. The School is in a Bolgatanga East District in the Upper East region of Ghana and lies along the BolgatangaBawku highway (Figure 1). This senior high school was chosen for the study through a random balloting procedure. The district has a population of about 28,641 [26] [27]. In terms of occupation, the residents of the locality were predominantly peasant farmers. The district shares boundaries with Tongo district to the South, Bolgatanga Municipal to the West, Nabdam district to the East, and Bongo District to the North [27].

\subsection{Study Population}

The target population for the study was all students in a Senior High school in the Upper East Region, Ghana. Participants in the study ranged from $14-19$ years in age and spanned through all classes in the school.

\subsection{Sampling and Sample Size}

Total enrolment in the school during the time of the study between August to December 2019 was 2081 students comprising 946 (45.5\%) males and 1135 (54.5\%) females. From the total enrolment, a proportionate sample of 329 was chosen using Krejcie and Morgan's [28] published tables for determining sample size.

A systematic random selection procedure was employed in selecting participants. The school had a total of 27 classrooms. Given the total number of students and gender disaggregation, a list of all students present at school during the survey was obtained from the school authorities, and after exclusion of thirty-one students who refused consent to participate in the study. From the list of all students who gave voluntary verbal consent to participate, a systematic but random selection of every $\mathrm{k}^{\text {th }}$ student was considered. Thus, from the normal-row seating

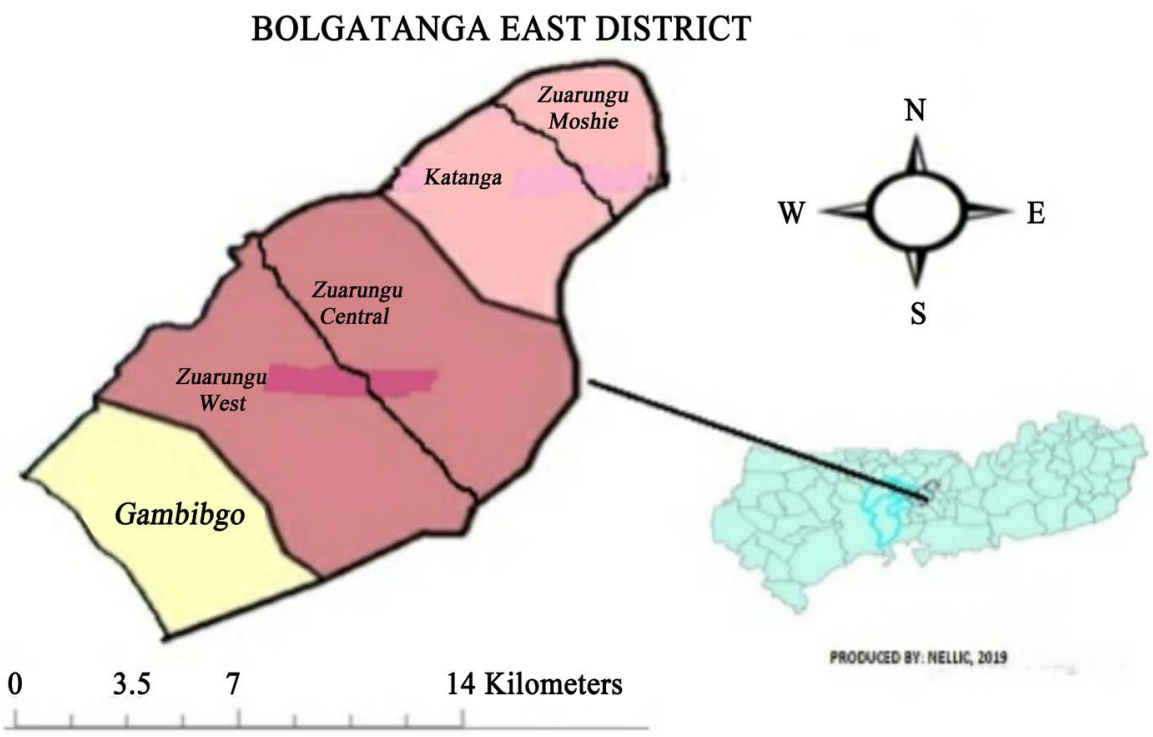

Figure 1. Map of Bolgatanga East District. 
arrangement in each class, every $6^{\text {th }}$ student was selected, until the final person in the $27^{\text {th }}$ class was considered. The procedure generated a total sample of 329 students, comprising 166 (50.5\%) males and 163 (49.5\%) females. Only 87 students were first-year students.

\subsection{Instrumentation}

A structured questionnaire was used in this study. These questions were developed from knowledge of current literature and the study contexts. The instruments were pilot tested with a sample of 57 students in a different senior high school in the same region. Questions were structured into six sections: socio-demographic information, sexuality education at home, sex education in the school, knowledge of students on sex education, contraceptive use among students, and the attitude of students towards sex education.

\subsection{Data Processing, Management, and Analysis}

All questionnaires were manually examined for completeness, then hand-coded and entered IBM SPSS (version 20) statistical software. Two research assistants double entered the data. Each member of the research team then independently compared the two data entries. Data completeness and correctness were ensured by running and comparing frequencies on each variable from the two data sets. This helped check incorrectly coded or entered data. All incorrectly coded/entered data were double-checked with raw data from the questionnaires, and all errors were discussed and resolved. A single database was then created and agreed upon before the data analysis commenced. Descriptive statistics were used to describe the crucial characteristics of participants. All variables were summarised into frequencies and proportions.

\subsection{Quality Control}

Three research assistants with a minimum undergraduate degree in the social sciences and public health fields who were proficient in the English Language were recruited and trained by the research team for a week on ethics in research, questionnaire administration, data integrity, and confidentiality issues of participants. The training also included interpretation of survey questions and data management. The research team comprised experts in quantitative research who ensured data collection followed the study design and that integrity processes were observed. The questionnaire was checked each day for completeness, and any uncompleted questionnaire was followed-up with the appropriate respondent before leaving the school.

\subsection{Ethical Issues}

Ethical approval was sought and obtained from the Navrongo Health Research Center's Review Board and Charles Sturt University Ethics Committee before the study began. 


\section{Participant recruitment}

The recruitment of participants was done entirely by the research team. This was to avoid participants feeling intimidated by teacher or instructor involvement in the selection and recruitment process. The purpose of the research was explained to the participants before asking for their consent to participate in the study. Ample opportunity was given to the participants to ask questions related to the procedures of the study. Written informed consent, signed or thumbprint was obtained from all participants, and participation was entirely voluntary. There were no direct benefits to participants. Participants were free to withdraw from the study at any time. The research participants were not required to provide information that was harmful to their studentship and academic work. The anonymity of the participants and school were protected during data analysis and publications by ensuring that no names and other identifying features are included.

\section{Results}

\subsection{Background Characteristics of Respondents}

Table 1 presents the sex disaggregation of participants. Out of the 329, males and females comprised of 50.5\% $(n=166)$ and $49.5 \%(n=163)$, respectively. Similarly, 191 respondents (58.1\%) were within the age group of 17 - 18 years, 138 respondents $(41.9 \%)$ were within the age bracket of 15 - 16 years, and no respondent was in the age group of 19-above years (Table 1). The senior high school educational systems are a three-year cycle that typically runs three terms per academic year. In terms of the year of study, the majority were in year two ( $n$ $=237,72 \%)$, followed by year ones $(n=87,26.00 \%)$. At the same time, just a few of the students consented to participate in the final year class due to preparations for final and external examinations (Table 1). Most participants were from the Business classes $(n=127,38.20)$, and Home Economics class $(n=69,21.00 \%)$.

\subsection{Students' Sources of Knowledge and Action Related to Knowledge}

Table 2 contains a Likert scale item on students' knowledge of sex education. Taking the results from individual item measures, many respondents ( $n=197$, $60.00 \%$ ) strongly disagreed that parents satisfactorily answer questions on their sexuality, with only $2 \%(n=7)$, who strongly agreed that parents answer the questions of adolescents, thoroughly. Males to female' differences in those who strongly opposed this assertion were insignificant (50.3\% versus $49.7 \%$ ).

Table 1. Sex distribution of respondents.

\begin{tabular}{ccc}
\hline Sex and age $(\mathrm{n}=\mathbf{3 2 9})$ & Frequency $(\mathrm{n})$ & Percent $(\%)$ \\
\hline Males & 166 & 50.50 \\
Females & 163 & 49.50 \\
$15-16$ & 138 & 41.90 \\
$17-18$ & 191 & 58.10 \\
\hline
\end{tabular}


Table 2. Students' sources of knowledge and action related to knowledge.

\begin{tabular}{|c|c|c|c|c|}
\hline Statement & $\mathrm{n}(\%)^{1}$ & $\begin{array}{l}\text { Males, } \\
\mathrm{n}(\%)^{2}\end{array}$ & $\begin{array}{c}\text { Females, } \\
\text { n }(\%)^{2}\end{array}$ & $\begin{array}{l}\text { Total, } \\
\text { n }(\%)^{2}\end{array}$ \\
\hline \multicolumn{5}{|c|}{ Parents answer the questions of their wards on their sexuality satisfactorily } \\
\hline Strongly disagree & $197(59.9)$ & $99(50.3)$ & $98(49.7)$ & $197(100)$ \\
\hline Disagree & $65(19.8)$ & $30(46.2)$ & $35(53.8)$ & $65(100)$ \\
\hline Agree & $60(18.2)$ & $37(61.7)$ & $23(38.3)$ & $60(100)$ \\
\hline Strongly agree & $7(2.1)$ & 0 & $7(100.0)$ & $7(100)$ \\
\hline \multicolumn{5}{|c|}{ Staying at home with my parents always gives me education on my sexuality } \\
\hline Strongly disagree & $87(26.4)$ & $35(40.2)$ & $52(59.8)$ & $87(100)$ \\
\hline disagree & $49(14.9)$ & $19(38.8)$ & $30(61.2)$ & $49(100)$ \\
\hline Agree & $143(43.5)$ & $83(53.0)$ & $60(42.0)$ & $143(100)$ \\
\hline Strongly agree & $50(15.2)$ & $29(58.0)$ & $21(42.0)$ & $50(100)$ \\
\hline \multicolumn{5}{|c|}{ Issues of my sexuality should be discussed at home with my parents at early stages } \\
\hline Strongly disagree & $171(52.0)$ & $79(46.2)$ & $92(53.8)$ & $171(100)$ \\
\hline Disagree & $51(15.5)$ & $22(43.1)$ & $29(56.9)$ & $51(100)$ \\
\hline Agree & $84(25.5)$ & $47(56.0)$ & $37(44.0)$ & $84(100)$ \\
\hline Strongly agree & $23(7.0)$ & $18(78.3)$ & $5(21.7)$ & $23(100)$ \\
\hline \multicolumn{5}{|c|}{ Knowledge of my parents on my relationship guides me to make better decisions } \\
\hline Strongly disagree & $143(43.5)$ & $53(37.1)$ & $90(62.9)$ & $143(100)$ \\
\hline Disagree & $80(24.3)$ & $49(61.2)$ & $31(38.8)$ & $80(100)$ \\
\hline Agree & $73(22.2)$ & $44(60.3)$ & $29(39.7)$ & $73(100)$ \\
\hline Strongly agree & $33(10.0)$ & $20(60.6)$ & $13(39.4)$ & $33(100)$ \\
\hline \multicolumn{5}{|c|}{ Developmental changes in my body shows that I am getting mature } \\
\hline Strongly disagree & $103(31.3)$ & $53(51.5)$ & $50(48.5)$ & $103(100)$ \\
\hline Disagree & $87(26.4)$ & $42(48.3)$ & $45(51.7)$ & $87(100)$ \\
\hline Agree & $108(32.8)$ & $57(52.8)$ & $51(47.2)$ & $108(100)$ \\
\hline Strongly agree & $31(9.4)$ & $14(45.2)$ & $17(54.8)$ & $31(100)$ \\
\hline \multicolumn{5}{|c|}{ My first developmental changes should be made known to my parents } \\
\hline Strongly disagree & $96(29.2)$ & $35(36.5)$ & $61(63.5)$ & $96(100)$ \\
\hline Disagree & $29(8.8)$ & $13(44.8)$ & $16(55.2)$ & $29(100)$ \\
\hline Agree & $155(47.1)$ & $88(56.8)$ & $67(43.2)$ & $155(100)$ \\
\hline Strongly agree & $49(14.9)$ & $30(61.2)$ & $19(38.8)$ & $49(100)$ \\
\hline \multicolumn{5}{|c|}{ My parents teach me how to guide myself against sexual pressures from the opposite sexes } \\
\hline Strongly disagree & $126(38.3)$ & $64(50.8)$ & $62(49.2)$ & $126(100)$ \\
\hline Disagree & $46(14.0)$ & $21(45.7)$ & $25(54.3)$ & $46(100)$ \\
\hline Agree & $136(41.3)$ & $76(55.9)$ & $60(44.1)$ & $136(100)$ \\
\hline Strongly agree & $21(6.4)$ & $5(23.8)$ & $16(76.2)$ & $21(100)$ \\
\hline \multicolumn{5}{|c|}{ Friends/peers can give better advice with regards my sexuality } \\
\hline Strongly disagree & $105(31.9)$ & $42(60.0)$ & $63(60.0)$ & $105(100)$ \\
\hline Disagree & $64(19.5)$ & $34(53.1)$ & $30(46.9)$ & $64(100)$ \\
\hline Agree & $107(32.5)$ & $62(57.9)$ & $45(42.1)$ & $107(100)$ \\
\hline Strongly agree & $53(16.1)$ & $28(52.8)$ & $25(47.2)$ & $53(100)$ \\
\hline
\end{tabular}




\section{Continued}

I practice abstinence in my past and present relationships

Strongly disagree

Disagree

Agree

Strongly agree

I prefer to discuss issues about my sexuality at school with my teacher

Strongly disagree

Disagree

Agree

Strongly agree

There are sex education programs in my school

Strongly disagree

Disagree

Agree

Strongly agree

Sex education programs should be organized every term in my school

Strongly disagree

Disagree

Agree

Strongly agree

I understand issues about sexuality taught in school better than at home

Strongly disagree

Disagree

Agree

Strongly agree

I sometimes visit my peers in school for some advices on issues about my relationships and sexual problems

Strongly disagree

Disagree

Agree

Strongly agree

Relationship and courtship are taught in other subjects in school

Strongly disagree

Disagree

Agree

Strongly agree

I understand what the teachers teach in school safe sex life

Strongly disagree

Disagree

Agree

Strongly agree

$\begin{array}{cccc}114(34.7) & 47(41.2) & 67(58.8) & 114(100) \\ 81(24.6) & 43(53.1) & 38(46.9) & 81(100) \\ 105(31.9) & 59(56.2) & 46(43.8) & 105(100) \\ 29(8.8) & 17(58.6) & 12(41.4) & 29(100)\end{array}$

$\begin{array}{cccc}161(48.9) & 68(42.2) & 93(57.8) & 161(100) \\ 57(17.3) & 34(59.6) & 23(40.4) & 57(100) \\ 85(25.8) & 52(61.2) & 33(38.8) & 85(100) \\ 26(7.9) & 12(46.2) & 14(53.8) & 26(100)\end{array}$

$136(41.3) \quad 63(46.3) \quad 73(53.7) \quad 136(100)$

$22(6.7) \quad 14(63.6) \quad 8(36.4) \quad 22(100)$

$148(45.0) \quad 78(52.7) \quad 70(47.3) \quad 148(100)$

$23(7.0) \quad 11(47.8) \quad 12(52.2) \quad 23(100)$

$\begin{array}{cccc}162(49.2) & 97(59.9) & 65(40.1) & 162(100) \\ 44(13.4) & 12(27.3) & 32(72.7) & 44(100) \\ 88(26.7) & 43(48.9) & 45(51.1) & 88(100) \\ 35(10.6) & 14(40.0) & 21(60.0) & 35(100) \\ & & & \\ & & & \\ 154(46.8) & 72(46.8) & 82(53.2) & 154(100) \\ 49(14.9) & 19(38.8) & 30(61.2) & 49(100) \\ 98(29.8) & 55(56.1) & 43(43.9) & 98(100) \\ 28(8.5) & 20(71.4) & 8(28.6) & 28(100)\end{array}$

$131(39.8) \quad 57(43.5) \quad 74(56.5) \quad 131(100)$

$86(26.1) \quad 48(55.8) \quad 38(44.2) \quad 86(100)$

$95(28.9) \quad 49(51.6) \quad 46(48.4) \quad 95(100)$

$17(5.2) \quad 12(70.6) \quad 5(29.4) \quad 17(100)$

$\begin{array}{cccc}125(38.0) & 68(54.4) & 57(45.6) & 125(100) \\ 88(26.7) & 40(45.5) & 48(54.5) & 88(100) \\ 99(30.1) & 52(52.5) & 47(47.5) & 99(100) \\ 17(5.2) & 6(35.3) & 11(64.7) & 17(100) \\ & & & \\ 161(48.9) & 91(56.5) & 70(43.5) & 161(100) \\ 49(14.9) & 21(42.9) & 28(57.1) & 49(100) \\ 93(28.3) & 41(44.1) & 52(55.9) & 93(100) \\ 26(7.9) & 13(50.0) & 13(50.0) & 26(100)\end{array}$




\section{Continued}

I get the opportunity to talk to my teachers every time about my sex life

$\begin{array}{lcccc}\text { Strongly disagree } & 132(40.1) & 61(46.2) & 71(53.8) & 132(100) \\ \text { Disagree } & 55(16.7) & 22(40.0) & 33(60.0) & 55(100) \\ \text { Agree } & 123(37.4) & 66(53.7) & 57(46.3) & 123(100) \\ \text { Strongly agree } & 19(5.8) & 17(89.5) & 2(10.5) & 19(100)\end{array}$

${ }^{1}$ Column percentages; ${ }^{2}$ Row percentages.

It was shown that the majority ( $\mathrm{n}=176,53.5 \%)$ strongly disagree with the notion that early sex can lead to unwanted pregnancies, with only $6.4 \%$ actively supporting the statement. Females were more strongly opposed to this assertion, although gender disparities were insignificant (49.4\% versus $50.6 \%$ ).

On answers concerning "parents answer the questions of their wards on their sexuality satisfactorily," most of the respondents $(n=197,59.9 \%)$ strongly disagreed with getting satisfactory responses. More females than males strongly agreed that parents satisfactorily addressed their concerns on sexuality.

Males more strongly agreed to this assertion that females (58.0\% versus $42.0 \%)$. Most students $(\mathrm{n}=155,47.1 \%)$ agreed that their first developmental changes (also known as puberty signs) should be disclosed to parents. However, more males than females agreed to let parents know about developmental changes (56.8\% versus $43.2 \%)$. More than half of the students $(\mathrm{n}=171,52 \%)$ strongly disagreed that "issues of my sexuality should be discussed at home with my parents at early stages." In contrast, only $7 \%$ strongly agreed that issues about their sexuality should be discussed at home with their parents.

Approximately $30 \%(n=99)$ agreed that they receive the counsel of their peers on sexuality while the majority $(\mathrm{n}=125,38 \%)$ strongly disagreed with obtaining the advice of peers on sexuality.

In the instructional design, students are often entreated by the educational management and parents to discuss issues of sexuality with teachers. The results show that the vast majority strongly disagree $(\mathrm{n}=161,48.9 \%)$ to this recommendation/policy, with more males than females strongly disagreeing with this perspective (57.8\% versus $42.2 \%)$. On "I understand issues about sexuality taught in school better than at home," just a small proportion strongly agree to the relevance of the lessons for their understanding (8.5\%). While the majority strongly disagree, more females (53.2\%) than males (46.8\%) strongly disagree that sexuality education taught in school gives them the required understanding. In terms of students who discussed issues of sexuality with teachers, $37.4 \%(n=123)$ agree that they received the opportunity anytime they desired to talk to their teachers about sexuality issues bordering on them (Table 2).

\subsection{Contraceptives Use among Students}

A series of questions sought to elicit students' views about contraceptive use among students in the school (Table 3). Specifically, most students $(n=136,41.3 \%)$ strongly disagreed that they had heard about contraceptives. More females than males strongly agreed to ever hearing about contraceptives (62.2 versus $37.8 \%$ ). 
Table 3. Contraceptive use among students.

\begin{tabular}{|c|c|c|c|c|}
\hline Statement & $\mathrm{n}(\%)^{1}$ & $\begin{array}{l}\text { Males, } \\
\mathrm{n}(\%)^{2}\end{array}$ & $\begin{array}{c}\text { Females, } \\
\text { n }(\%)^{2}\end{array}$ & $\begin{array}{l}\text { Total } \\
\mathrm{n}(\%)^{2}\end{array}$ \\
\hline \multicolumn{5}{|l|}{ I have heard about contraceptives } \\
\hline Strongly disagree & $136(41.3)$ & $82(60.3)$ & $54(39.7)$ & $136(100)$ \\
\hline Disagree & $42(12.8)$ & $20(47.6)$ & $22(52.4)$ & $42(100)$ \\
\hline Agree & $114(34.7)$ & $50(43.9)$ & $64(56.1)$ & $114(100)$ \\
\hline Strongly agree & $37(11.2)$ & $14(37.8)$ & $23(62.2)$ & $37(100)$ \\
\hline \multicolumn{5}{|l|}{ I have used contraceptives before } \\
\hline Strongly disagree & $144(43.8)$ & $79(54.9)$ & $65(45.1)$ & $144(100)$ \\
\hline Disagree & $57(17.3)$ & $21(36.8)$ & $36(63.2)$ & $57(100)$ \\
\hline Agree & $90(27.4)$ & $43(47.8)$ & $47(52.2)$ & $90(100)$ \\
\hline Strongly agree & $38(11.6)$ & $23(60.5)$ & $15(39.5)$ & $38(100)$ \\
\hline \multicolumn{5}{|c|}{ I know that coitus interruptus method is a contraceptive method } \\
\hline Strongly disagree & $120(36.5)$ & $68(56.7)$ & $52(43.3)$ & $120(100)$ \\
\hline Disagree & $48(14.6)$ & $28(58.3)$ & $20(41.7)$ & $48(100)$ \\
\hline Agree & $105(31.9)$ & $43(41.0)$ & $62(59.0)$ & $105(100)$ \\
\hline Strongly agree & $56(17.0)$ & $27(48.2)$ & $29(51.8)$ & $56(100)$ \\
\hline \multicolumn{5}{|l|}{ I seek advice from peers on contraceptive use } \\
\hline Strongly disagree & $117(35.6)$ & $55(47.0)$ & $62(53.0)$ & $117(100)$ \\
\hline Disagree & $43(13.1)$ & $19(44.2)$ & $24(55.8)$ & $43(100)$ \\
\hline Agree & $135(41.0)$ & $77(57.0)$ & $58(43.0)$ & $135(100)$ \\
\hline Strongly agree & $34(10.3)$ & $15(44.1)$ & $19(55.9)$ & $34(100)$ \\
\hline \multicolumn{5}{|c|}{ I know condom as the most commonly used contraceptive among my peers } \\
\hline Strongly disagree & $139(42.2)$ & $80(57.6)$ & $59(42.4)$ & $139(100)$ \\
\hline Disagree & $54(16.4)$ & $20(37.0)$ & $34(63.0)$ & $54(100)$ \\
\hline Agree & $106(32.2)$ & $49(46.2)$ & $57(53.8)$ & $106(100)$ \\
\hline Strongly agree & $30(9.1)$ & $17(56.7)$ & $13(43.3)$ & $30(100)$ \\
\hline \multicolumn{5}{|c|}{ I know that sperm can live in a woman's body for up to three days } \\
\hline Strongly disagree & $68(20.7)$ & $50(73.5)$ & $18(26.5)$ & $68(100)$ \\
\hline Disagree & $69(21.0)$ & $25(36.2)$ & $44(63.8)$ & $69(100)$ \\
\hline Agree & $123(37.4)$ & $65(52.8)$ & $58(47.2)$ & $123(100)$ \\
\hline Strongly agree & $69(21.0)$ & $26(37.7)$ & $43(62.3)$ & $69(100)$ \\
\hline \multicolumn{5}{|c|}{ A female urinating immediately after sex can sometimes prevent pregnancy } \\
\hline Strongly disagree & $106(32.2)$ & $56(52.8)$ & $50(47.2)$ & $106(100)$ \\
\hline Disagree & $50(15.2)$ & $26(52.0)$ & $24(48.0)$ & $50(100)$ \\
\hline Agree & $140(42.6)$ & $69(49.3)$ & $71(50.7)$ & $140(100)$ \\
\hline Strongly agree & $33(10.0)$ & $15(45.5)$ & $18(54.5)$ & $33(100)$ \\
\hline \multicolumn{5}{|c|}{ A female who has sex only occasionally does not need any birth control method } \\
\hline Strongly disagree & $123(37.4)$ & $76(61.8)$ & $47(38.2)$ & $123(100)$ \\
\hline Disagree & $39(11.9)$ & $9(23.1)$ & $30(76.9)$ & $39(100)$ \\
\hline Agree & $101(30.7)$ & $53(52.5)$ & $48(47.5)$ & $101(100)$ \\
\hline Strongly agree & $66(20.1)$ & $28(42.4)$ & $38(57.6)$ & $66(100)$ \\
\hline
\end{tabular}

${ }^{1}$ Column percentages; ${ }^{2}$ Row percentages. 
In terms of contraceptive usage, many of the students $(\mathrm{n}=144,43.8 \%)$ said they had never used contraceptives, compared to the much smaller number who strongly agreed that they had used contraception in the past $(\mathrm{n}=37,11.2 \%)$. Among students $(\mathrm{n}=38,11.6 \%)$ who agreed that they had used contraceptives, the proportion of males to females was $11.6 \%$ and $39.5 \%$, respectively.

Approximately $41.0 \%(n=135)$ of the students agreed that they sought the counsel of their peers regarding contraceptive use. This was more common with males than females; $57.0 \%$ versus $43.0 \%$. A significant majority of participants (n $=140,42.6 \%)$ agreed $(\mathrm{n}=140,42.6 \%)$ or strongly agreed $(10 \%)$ that a female urinating immediately after sexual intercourse can sometimes prevent pregnancy. It was found that many females (50.7\%) agreed to this assertion.

The statement that "a female who has sex only once in a while does not need any birth control method" was strongly disagreed with by a large proportion of students $(\mathrm{n}=123,37.4 \%)$. Among the students who strongly agree with this perception among students $(n=60,20.1 \%)$, more females strongly agreed to this assertion than males $(57.6 \%$ versus $42.4 \%)$. Similarly, about $42 \%(\mathrm{n}=139)$ of participants strongly disagree that condom is the most commonly used contraceptive commodity among the student population. In contrast, a small proportion $(\mathrm{n}=30,9.1 \%)$ strongly agree that condom was the most widely used contraceptive among their peers.

\subsection{Participant Actions Regarding Sexuality}

We also sought to understand the attitude of students towards sex education (Table 4). The results have shown that reporting "my first developmental changes to my parents and teachers" was strongly disagreed with by most of the respondents $(\mathrm{n}=150,45.6 \%)$. However, quite a significant proportion also agreed $(\mathrm{n}=101,30.7 \%)$. For students who agreed with this statement, more males were in support of it than females (55.4\% versus $44.6 \%$ ).

The statement "seek advice on contraceptive use from health personnel" was strongly disagreed with by a large proportion (41.6\%). Females were more likely to strongly oppose the recommendation (52.6\% versus $47.4 \%$ of males). However, a significant proportion of participants agreed that advice on contraceptive use should be sought from health personnel $(\mathrm{n}=126,38.3 \%)$. More males $(51.6 \%)$ agreed to the assertion than females $(48.4 \%)$. We also observed that many students $(\mathrm{n}=151,45.9 \%)$ strongly agreed that "my partner should use a contraceptive during sexual intercourse." Males to females' dichotomy was $47.7 \%$ to $52.3 \%$, respectively. However, only $10.6 \%(n=35)$ strongly agreed to contraceptive use during sexual intercourse.

Most students ( $\mathrm{n}=134,40.7 \%)$ strongly disagreed with the statement, "teachers can give the best of counseling about issues of my sexuality." More males (n $=71,53.0 \%)$ than females $(\mathrm{n}=63,47.0 \%)$ strongly oppose that assertion. Among the few students who agreed $(n=95,28.9 \%)$ or strongly agreed $(n=23$, $7.0 \%)$, females were more receptive to that perspective than males $(60.0 \%$ versus 40.0\%) (Table 4). 
Table 4. Attitude of adolescent students towards their sexuality.

\begin{tabular}{|c|c|c|c|c|}
\hline Statement & $\mathrm{n}(\%)^{1}$ & $\begin{array}{l}\text { Males, } \\
\text { n (\%) }\end{array}$ & $\begin{array}{c}\text { Females, } \\
\mathrm{n}(\%)^{2}\end{array}$ & $\begin{array}{l}\text { Total } \\
\text { n }(\%)^{2}\end{array}$ \\
\hline \multicolumn{5}{|c|}{ Report my first developmental changes to my parents and teachers } \\
\hline Strongly disagree & $150(45.6)$ & $68(45.3)$ & $82(54.7)$ & $150(100)$ \\
\hline Disagree & $52(15.8)$ & $24(46.2)$ & $28(53.8)$ & $52(100)$ \\
\hline Agree & $101(30.7)$ & $56(55.4)$ & $45(44.6)$ & $101(100)$ \\
\hline Strongly agree & $26(7.9)$ & $18(69.2)$ & $8(30.8)$ & $26(100)$ \\
\hline \multicolumn{5}{|c|}{ Seek advice on contraceptive use from health personnel } \\
\hline Strongly disagree & $137(41.6)$ & $65(47.4)$ & $72(52.6)$ & $137(100)$ \\
\hline Disagree & $33(10.0)$ & $20(60.6)$ & $13(39.4)$ & $33(100)$ \\
\hline Agree & $126(38.3)$ & $65(51.6)$ & $61(48.4)$ & $126(100)$ \\
\hline Strongly agree & $33(10.0)$ & $16(48.5)$ & $17(51.5)$ & $33(100)$ \\
\hline \multicolumn{5}{|c|}{ My partner should use contraceptive during sexual intercourse } \\
\hline Strongly disagree & $151(45.9)$ & $72(47.7)$ & $79(52.3)$ & $151(100)$ \\
\hline Disagree & $45(13.7)$ & $32(71.1)$ & $13(28.9)$ & $45(100)$ \\
\hline Agree & $98(29.8)$ & $49(50.0)$ & $49(50.0)$ & $98(100)$ \\
\hline Strongly agree & $35(10.6)$ & $13(37.1)$ & $22(62.9)$ & $35(100)$ \\
\hline \multicolumn{5}{|c|}{ Early sex can lead one to get unwanted pregnancies } \\
\hline Strongly disagree & $176(53.5)$ & $87(49.4)$ & $89(50.6)$ & $176(100)$ \\
\hline Disagree & $54(16.4)$ & $37(68.5)$ & $17(31.5)$ & $54(100)$ \\
\hline Agree & $78(23.7)$ & $36(46.2)$ & $42(53.8)$ & $78(100)$ \\
\hline Strongly agree & $21(6.4)$ & $6(28.6)$ & $15(71.4)$ & $21(100)$ \\
\hline \multicolumn{5}{|c|}{ Discussing issues of my sexuality with my friends keeps me up to date } \\
\hline Strongly disagree & $141(42.9)$ & $69(48.9)$ & $72(51.1)$ & $141(100)$ \\
\hline Disagree & $46(14.0)$ & $22(47.8)$ & $24(52.2)$ & $46(100)$ \\
\hline Agree & $110(33.4)$ & $60(54.5)$ & $50(45.5)$ & $110(100)$ \\
\hline Strongly agree & $32(9.7)$ & $15(46.9)$ & $17(53.1)$ & $32(100)$ \\
\hline \multicolumn{5}{|c|}{ Teachers can give the best of counselling about issues of my sexuality } \\
\hline Strongly disagree & $134(40.7)$ & $71(53.0)$ & $63(47.0)$ & $134(100)$ \\
\hline Disagree & $77(23.4)$ & $43(55.8)$ & $34(44.2)$ & $77(100)$ \\
\hline Agree & $95(28.9)$ & $38(40.0)$ & $57(60.0)$ & $95(100)$ \\
\hline Strongly agree & $23(7.0)$ & $14(60.9)$ & $9(39.1)$ & $23(100)$ \\
\hline \multicolumn{5}{|c|}{ Parents should be aware of their ward's relationships } \\
\hline Strongly disagree & $115(35.0)$ & $54(47.0)$ & $61(53.0)$ & $115(100)$ \\
\hline Disagree & $83(25.2)$ & $42(50.6)$ & $41(49.4)$ & $83(100)$ \\
\hline Agree & $107(32.5)$ & $57(53.3)$ & $50(46.7)$ & $107(100)$ \\
\hline Strongly agree & $24(7.3)$ & $13(54.2)$ & $11(45.8)$ & $24(100)$ \\
\hline \multicolumn{5}{|c|}{ Abstinence is the best in avoiding unwanted pregnancies } \\
\hline Strongly disagree & $142(43.2)$ & $72(50.7)$ & $70(49.3)$ & $142(100)$ \\
\hline Disagree & $49(14.9)$ & $22(44.9)$ & $27(55.1)$ & $49(100)$ \\
\hline Agree & $108(32.8)$ & $61(56.5)$ & $47(43.5)$ & $108(100)$ \\
\hline Strongly agree & $30(9.1)$ & $11(36.7)$ & $19(63.3)$ & $30(100)$ \\
\hline
\end{tabular}




\section{Continued}

\begin{tabular}{|c|c|c|c|c|}
\hline Strongly disagree & $135(41.0)$ & $72(53.3)$ & $63(46.7)$ & $135(100)$ \\
\hline Disagree & $50(15.2)$ & $24(48.0)$ & $26(52.0)$ & $50(100)$ \\
\hline Agree & $111(33.7)$ & $53(47.7)$ & $58(52.3)$ & $111(100)$ \\
\hline Strongly agree & $33(10.0)$ & 17 (51.5) & $16(48.5)$ & $33(100)$ \\
\hline
\end{tabular}

${ }^{1}$ Column percentages; ${ }^{2}$ Row percentages.

\section{Discussion}

Three critical perspectives on sex education were described: knowledge, contraception, and attitude towards sex education.

\subsection{Sex Education at School and Home}

From the four-point scale responses, it was found that most students do not believe that early sex can lead to unwanted pregnancies. Also, we found that considerable variation in students' preferred sources of information about sexual health information, thus, from peers, parents, teachers or health professionals, and the perceived value of each of these sources. Arguably, parents generally are more knowledgeable and more experienced in issues regarding sexuality than their adolescent. However, the findings demonstrate that most students still prefer to discuss related sexual matters with their peers rather than with their parents and teachers. This is reflected in the results showing that most students felt more secure in the company of their peers concerning issues about their sexuality (49\%) compared to their parents, with $80 \%$ of respondents who strongly felt that their parents did not give their questions satisfactory explanations. It is not surprising that the participants rely more on their peers for information and advice once parents would not be candid enough and answer their questions satisfactorily. Literature suggests that for adolescents to gain adequate knowledge of sexuality, it is ideal that parents respond to questions posed by their children satisfactorily [29]. These findings are consistent with previous studies in Ghana and similar locations [12] [14]. The adolescent literature has shown that most adolescents feel comfortable to share issues about their sexuality with their peers rather than with their parents [14] [25], in addition to sourcing information from the internet [6] [9] [24].

Participants' preferences for obtaining information and advice regarding sexuality from peers is also reflected in their views regarding the sharing of signs of puberty with parents, and parents' lack of willingness to offer guidance concerning sexual pressure within relationships. In most instances, when adolescent sees any change with regards to their morphology, reporting to parents, peers, teachers, and health personnel have shown to help reduce overall adverse outcomes and stigma [5] [18] [20]. Reporting these changes to the parents are mostly recommended [25]. The study found that $47 \%$ of adolescents agree that their first developmental change should be reported to their parents, whereas $29 \%$ indicated that they do not actively agree to sharing early puberty signs with parents. In the recent past, parents, especially mothers, were significantly involved in guiding 
their adolescent females through puberty [3] [17] [18] [30]. Females will naturally resort to their mothers while the males will resort to their father.

In this study, $41 \%$ of the participants agreed, $38 \%$ strongly disagreed, and only $6 \%$ agreed that their parents' guide them against sexual pressures from the opposite sexes. The lack of information and support from parents regarding sexuality is common in most African settings talking about sex, and its related topics are seen by most parents to be against their personal and community moral values, so they will always shy away [2] [10]. Tendencies are that children will not enjoy responsible parenting to prepare wards against sexual pressures from the opposite sex adequately [10].

Similarly, about $49 \%$ strongly opposed the suggestion to share issues of school children's sexuality matters with schoolteachers. From the literature in Ghana, it has shown that adolescents who are pressured by the opposite sex to engage in sexual activity may not inform teachers to prevent being tagged "bad" [20]. However, this finding contrasts with those of previous studies in Ghana, Zambia and other African nations [4] [14] [18] which suggests that a large proportion of adolescents (about 77\%) were "rather comfortable" disclosing and discussing matters of their sexuality with teachers [4] [18] in comparison to parents.

Both the teachers and students alike make do with subjects that have relevant education on the sex they can get from reproductive health topics in the reproductive system in biology and selected Family Life topics in the "Social Studies" subject [14] [31]. However, we found that about $41 \%$ of our participants strongly disagree that there are no sex education programs in the schools, which confirms previous findings in Ghana [31]. Previous researchers have observed that there were no explicit sex education programs in the Ghanaian school system, which has significant implications for Ghanaians in a globalised environment [14] [31].

It is apparent that even if sex education is being provided in schools, the content (assuming it is accurate) is not being understood by students. On students' views on the fact that early sexual intercourse can lead to unwanted pregnancies, approximately $11 \%$ and $22 \%$ strongly agree and agree, respectively. The majority (41\%) strongly disagree that early sexual intercourse was the risk of unwanted pregnancies. This belief is potentially one of the main reasons adolescent/teenage pregnancy is at an all-time in Ghana, with, on average, one in ten adolescents from 15 - 19 years beginning childbearing in the urban areas, and two in ten adolescents in the rural communities [18] [32]. This may be attributable to inadequate education on sexual activities and ways of preventing such behaviours. Recent adolescent literature has widely shown that providing inadequate information about sexuality is one means of facilitating the sexual exploitation of children and adolescents [16] [18] [33] [34].

\subsection{Understanding and Use of Contraceptives and Other Means of Pregnancy Prevention}

Lack of accurate information (or misunderstanding of the information given) about contraceptives and misperceptions about other ways to prevent pregnancies 
is apparent in participant responses. This has resulted in risky sexual behaviours, significant numbers of teenage pregnancies and terminations, and transmission of diseases.

On contraceptive use by students, the vast majority (44\%) strongly disagree with using it, while just about $12 \%$ strongly agree to the use of contraceptive during sexual intercourse. When participants were asked if a condom was the most commonly used contraceptive among their peers, $42 \%$ strongly disagreed, while 9\% strongly agreed that condom is the most frequently used contraceptive. The contraceptive acceptance rate among adolescent students in this study is lower than those reported in a previous study in Central Ghana [35]. The study reported that $24.0 \%$ among females and $39.0 \%$ among males was reported for married adolescents while $18.0 \%$ and $27.0 \%$ of adolescent males and females, respectively, used contraceptives (particularly condoms) during sexual encounters [35]. Previous research in the Upper West Region of Ghana has shown that even adult men, including opinion leaders, resisted women's use of contraceptives [36] [37]. Religious beliefs and cultural pervasion in Ghana portray access to contraceptives among unmarried adolescents, including school children, as "bad" boy or girl practices. Similarly, a previous study in the Upper West Region of Ghana involving adolescents and non-adolescent mothers and a host of community members, showed that contraceptive use in adult women was also strongly discouraged in some locations and indeed by some men [36] [37].

Inadequate/inaccurate information from peers and lack of information from parents, teachers, and health professionals contributes to the non-usage of contraceptives. The findings agree with the existing knowledge. Previous studies have reported myths on preventing pregnancy including the woman shaking herself and jumping up seven times; urinating immediately after the sexual encounter: having sex in water, drinking two liters of Mountain Dew before sex to lower sperm count, and females being on top during sexual encounters [22] [38].

The results showed significant misconceptions about contraceptive efficacy. After sexual intercourse, the sperms can stay in the fallopian tube for up to a maximum of 72 hours. This can fertilize the ovum when it is released [35]. Knowledge of this helps the adolescent to understand the menstrual cycle better and hence take precautionary measures.

In this study, the finding revealed most respondents held perceptions concerning adolescent sexual health service utilisation which corroborates with previous studies [37]. This was indicated in $43 \%$ of respondents agreed, $32 \%$ strongly disagreed, $15 \%$ disagree, and only $10 \%$ strongly agree to this perspective. The finding could be attributed to the cultural background of the majority of the respondents who were from the Northern parts of Ghana, where illiteracy is still on the rise. About $37 \%$ of respondents strongly disagree that having sex once in a while does not need any birth control method suggesting that these young adolescents can indulge in very dangerous sexual behaviours without having any protection based on the perception that sex once in a while does not need any contraceptive method(s). 
The finding suggests a change among adolescents, which corroborates previous findings in Ghana and similar low and middle-income countries [4] [22] [35]. Consequently, we discovered that about $41.6 \%$ of respondents strongly agree to seek peers' counsel on the use of contraceptives.

These misunderstandings and use of peers as primary sources of information regarding the prevention of pregnancy have persisted over the years among senior high school students in Ghana [19] [20] [23], showing a significant gap in knowledge, relevant sensitization needs and access to contraceptive services by the school youth. This is contrary to the long-established principle that knowledge about an issue presents a higher chance of making an informed decision [12] [33]. Further, contrary to previous research in Ghana, Mozambique and Zambia found that students desired education on contraceptives as well as more general discussions on sexual matters from health personnel [4] [23] [25]. In Tanzania, it was reported that $44.8 \%$ of adolescents receive information on their sexuality from their friends and peers [39].

\subsection{Implications and Contribution of the Manuscript}

The Ghana Health Service and School management committees have school health clubs and "adolescent corners," some of which were formed and resourced by non-governmental organizations. The clubs could be revamped and resourced with relevant personnel to educate students on sexuality, sexually transmitted diseases, and contraception, which could positively reflect students' sexual life.

\subsection{Limitations of the Study}

We acknowledge that the findings may not reflect the views of all students in the geographical area. However, given that students were randomly chosen for the study, adolescent perspectives found in the study would be reliable in addressing and designing adolescent sexual health education campaigns.

Further, religious pluralism in Ghana may account for students' perspectives on some statements, especially on contraceptive use and sexual practices is a potential limitation of the study. Thus, it is possible that more adolescents are using contraceptives but did not want to share this information in the survey because of the religious and other cultural beliefs attached to contraceptive use.

\section{Conclusions}

Peer influence has been a significant disruptor of sex education among adolescents. These adolescents are young individuals who would always want to experiment with everything they have learned from their peers or would receive pressure from their peers to experiment with what they have tested and experienced.

Peers from this study, are not disrupting sex education. Sex education is limited or non-existent from other "ideal" sources, so they are alternatively filling the gap for themselves. The future concerns are how adolescents would likely listen to and follow the advice of their parents, even if the parents are willing to 
discuss sexuality openly. Perhaps there are specific cultural reasons (e.g., respect for persons older than them) why they are likely to follow the advice of parents. The adequacy and relevance of information received from peers cannot be guaranteed; the chances are that wrong information may be provided these adolescents with huge implications on indulgence on risky reproductive and sexual health choices. Therefore, there is a call for the government and parents to intervene by helping educational educations develop holistic and friendly packages to increase children's sexual and reproductive health lifestyles.

From the perspectives of students, the institutional and family approach may potentially account for the information deficit. In most families in Ghana, the majority of parents and teachers feel that sexual issues should not be too openly discussed with these students because they would learn to become promiscuous sooner than later. Given the number of adolescents who are sexually active and who lack understanding of some of the basic facts related to contraception, based on the results of the survey, the contemporary adolescent sexual health education approach is failing. Thus, we suggest that an alternative approach be developed, not only focused on traditional potential sources of information (family and teachers) but also sources of information favoured by adolescents-their peers and online, and teachers.

The home as a unit of socialization must step-up to its mandate of helping the future generation understand the concerns embedded in sexual life by equipping them with the required understanding of sexuality so they could harmoniously exist in the global community.

\section{Acknowledgements}

We are indebted to the staff and management of the Senior High School for giving us access to, and for their help during the study.

\section{Conflicts of Interest}

The authors declare no conflicts of interest regarding the publication of this paper.

\section{References}

[1] GDHS: Ghana Statistical Service (GSS), Ghana Health Services (GHS), ICF Macro (2009) Ghana Demographic and Health Survey 2008. GSS, GHS, ICF Macro, Accra.

[2] Aninanya, G.A., Debpuur, C.Y., Awine, T., Williams, J.E., Hodgson, A. and Howard, N. (2015) Effects of an Adolescent Sexual and Reproductive Health Intervention on Health Service Usage by Young People in Northern Ghana: A Community-Randomised Trial. PLoS ONE, 10, e0125267. https://doi.org/10.1371/journal.pone.0125267

[3] Weaver, H., Smith, G. and Kippax, S. (2005) School-Based Sex Education Policies and Indicators of Sexual Health among Young People: A Comparison of the Netherlands, France, Australia and the United States. Sex Education, 5, 171-188. https://doi.org/10.1080/14681810500038889 
[4] Zulu, J.M., Blystad, A., Haaland, M.E., Michelo, C., Haukanes, H. and Moland, K.M. (2019) Why Teach Sexuality Education in School? Teacher Discretion in Implementing Comprehensive Sexuality Education in Rural Zambia. International Journal for Equity in Health, 18, 116. https://doi.org/10.1186/s12939-019-1023-1

[5] Baams, L., Overbeek, G., van de Bongardt, D., Reitz, E., Dubas, J.S. and van Aken, M.A. (2015) Adolescents' and Their Friends' Sexual Behavior and Intention: Selection Effects of Personality Dimensions. Journal of Research in Personality, 54, 2-12. https://doi.org/10.1016/j.jrp.2014.07.009

[6] Senanayake, P., Nott, J.H. and Faulkner, K.M. (2001) Adolescent Sexual and Reproductive Health: The Challenge for Society. Human Fertility, 4, 117-122. https://doi.org/10.1080/1464727012000199401

[7] UNAIDS (2000) Epidemiological Fact Sheet on HIV/AIDS and Sexually Transmitted Infections, 2002 Update. Joint United Nations Program on HIV AIDS, Geneva.

[8] UNAIDS (2017) Joint United Nations Programme on HIV/AIDS (UNAIDS) Data. Global and Regional Data HIV/SIDA, 12-16.

[9] Staff U (2011) The State of the World's Children 2011 Executive Summary: Adolescence an Age of Opportunity.

[10] GHS (2015) Adolescent Health Service Policy and Strategy (2016-2020). Ghana Health Service, Accra.

[11] UNFPA (1994) ICPD Programme of Action.

[12] Hagan, J.E. and Buxton, C. (2012) Contraceptive Knowledge, Perceptions and Use among Adolescents in Selected Senior High Schools in the Central Region of Ghana. Journal of Sociological Research, 3, 170-180. https://doi.org/10.5296/jsr.v3i2.2311

[13] Rominski, S.D. and Stephenson, R. (2019) Toward a New Definition of Unmet Need for Contraception. Studies in Family Planning, 50, 195-198. https://doi.org/10.1111/sifp.12084

[14] Panchaud, C., Keogh, S.C., Stillman, M., Awusabo-Asare, K., Motta, A., Sidze, E. and Monzón, A.S. (2019) Towards Comprehensive Sexuality Education: A Comparative Analysis of the Policy Environment Surrounding School-Based Sexuality Education in Ghana, Peru, Kenya and Guatemala. Sex Education, 19, 277-296. https://doi.org/10.1080/14681811.2018.1533460

[15] HIV/AIDS JUNPo (2006) Report on the Global AIDS Epidemic. UNAIDS, Geneva.

[16] Ogbada, A.E. (2013) Determinants of Risky Sexual Behaviour among Senior High School Students, in La Dade-Kotopon Municipality. University of Ghana, Accra.

[17] Bansal, R. and Mehra, M. (1998) Adolescent Girls: An Emerging Priority. Indian Journal of Public Health, 42, 1.

[18] Bingenheimer, J.B., Asante, E. and Ahiadeke, C. (2015) Peer Influences on Sexual Activity among Adolescents in Ghana. Studies in Family Planning, 46, 1-19. https://doi.org/10.1111/j.1728-4465.2015.00012.x

[19] Abakah, B. (2015) Sex Education and Its Influence on Sexual Behavior in the West African Senior High School in the Greater Accra Region. University of Ghana, Accra.

[20] Adoboah, E. (2012) Sexual Behaviour of In-School Early Adolescents: A Case Study in New Juaben Municipality. University of Cape Coast, Cape Coast.

[21] Cantey, N.I., Bland, R., Mack, L.R. and Joy-Davis, D. (2013) Historically Black Colleges and Universities: Sustaining a Culture of Excellence in the Twenty-First Century. Journal of African American Studies, 17, 142-153. https://doi.org/10.1007/s12111-011-9191-0 
[22] Adongo, P.B., Tabong, P.T.-N., Azongo, T.B., Phillips, J.F., Sheff, M.C., Stone, A.E. and Tapsoba, P. (2014) A Comparative Qualitative Study of Misconceptions Associated with Contraceptive Use in Southern and Northern Ghana. Frontiers in Public Health, 2, 137. https://doi.org/10.3389/fpubh.2014.00137

[23] Asampong, E., Osafo, J., Bingenheimer, J.B. and Ahiadeke, C. (2013) Adolescents and Parents' Perceptions of Best Time for Sex and Sexual Communications from Two Communities in the Eastern and Volta Regions of Ghana: Implications for HIV and AIDS Education. BMC International Health and Human Rights, 13, 40. https://doi.org/10.1186/1472-698X-13-40

[24] Kwankye, S.O. and Augustt, E. (2007) Media Exposure and Reproductive Health Behaviour among Young Females in Ghana. African Population Studies, 22, 77-106. https://doi.org/10.11564/22-2-330

[25] Miedema, E. and Oduro, G.Y. (2017) Sexuality Education in Ghana and Mozambique: An Examination of Colonising Assemblages Informing School-Based Sexuality Education Initiatives. In: The Palgrave Handbook of Sexuality Education, Springer, Berlin, 69-93. https://doi.org/10.1057/978-1-137-40033-8_4

[26] Adanse, J., Atinga, A. and Yamga, L. (2017) Investigating the Health and Safety Measures in the Kitchen: A Study of Some Selected Second Cycle Institutions in Bolgatanga Municipality of Ghana. Journal of Tourism and Hospitality Management, 5, 45-55. https://doi.org/10.15640/jthm.v5n2a5

[27] GSS (2013) 2010 Population and Housing Census. GoG, Accra, 430.

[28] Krejcie, R.V. and Morgan, D.W. (1970) Determining Sample Size for Research Activities. Educational and Psychological Measurement, 30, 607-610. https://doi.org/10.1177/001316447003000308

[29] Johnson, A.L., Collins, L.K., Villanti, A.C., Pearson, J.L. and Niaura, R.S. (2018) Patterns of Nicotine and Tobacco Product Use in Youth and Young Adults in the United States, 2011-2015. Nicotine \& Tobacco Research, 20, S48-S54. https://doi.org/10.1093/ntr/nty018

[30] Singh, S., Sedgh, G. and Hussain, R. (2010) Unintended Pregnancy: Worldwide Levels, Trends, and Outcomes. Studies in Family Planning, 41, 241-250. https://doi.org/10.1111/j.1728-4465.2010.00250.x

[31] Asiedu, S., Blankson, E., Abane, A., Owusu, S., Blankson, E. and Abane, A. (2014) Women's Health and Action Research Centre (WHARC) Sexual and Reproductive Health Education among Dressmakers and Hairdressers in the Assin South District of Ghana.

[32] Ganle, J.K., Obeng, B., Yeboah, J.Y., Tagoe-Darko, E. and Mensah, C.M. (2016) Disparities in Abortion Experience and Access to Safe Abortion Services in Ghana: Evidence from a Retrospective Survey. African Journal of Reproductive Health, 20, 43-52. https://doi.org/10.29063/ajrh2016/v20i2.5

[33] Kaestle, C.E., Halpern, C.T., Miller, W.C. and Ford, C.A. (2005) Young Age at First Sexual Intercourse and Sexually Transmitted Infections in Adolescents and Young Adults. American Journal of Epidemiology, 161, 774-780. https://doi.org/10.1093/aje/kwi095

[34] Kumi-Kyereme, A., Awusabo-Asare, K. and Darteh, E.K. (2014) Attitudes of Gatekeepers towards Reproductive Health in Ghana. African Journal of Reproductive Health, 18, 142-153.

[35] Boamah, E.A., Asante, K.P., Mahama, E., Grace, M., Ayipah, E.K., Adeniji, E. and Owusu-Agyei, S. (2014) Use of Contraceptives among Adolescents in Kintampo, 
Ghana: A Cross-Sectional Study. Open Access Journal of Contraception, 5, 7. https://doi.org/10.2147/OAJC.S56485

[36] Sumankuuro, J. (2018) Paradoxes of Factors Influencing Maternal Health Outcomes in Rural Northern Ghana. Charles Sturt University, Dubbo.

[37] Sumankuuro, J., Crockett, J. and Wang, S. (2018) Perceived Barriers to Maternal and Newborn Health Services Delivery: A Qualitative Study of Health Workers and Community Members in Low and Middle-Income Settings. BMJ Open, 8, e021223. https://doi.org/10.1136/bmjopen-2017-021223

[38] Elia, J.P. and Eliason, M.J. (2009) Values-Free Sexuality Education: Myth or Reality. In: Sexuality Education: Past, Present, and Future, Praeger, Westport, Vol. 1, 174-198.

[39] Sweya, M.N., Msuya, S.E., Mahande, M.J. and Manongi, R. (2016) Contraceptive Knowledge, Sexual Behavior, and Factors Associated with Contraceptive Use among Female Undergraduate University Students in Kilimanjaro Region in Tanzania. Adolescent Health, Medicine and Therapeutics, 7, 109.

https://doi.org/10.2147/AHMT.S108531 